\title{
Alterations in neuroblastoma ganglioside synthesis by induction of GDIb synthase by retinoic acid
}

\author{
S Hettmer ${ }^{1,3}$, R McCarter ${ }^{2,3}$, S Ladisch ${ }^{1,3}$ and K Kaucic ${ }^{*, 1,3}$ \\ 'Glycobiology Program, Center for Cancer and Immunology Research, Children's National Medical Center, II I Michigan Avenue, NW, Washington DC \\ 200 I O, USA; 'Biostatistics and Informatics Unit, Center for Health Services and Community Research, Children's National Medical Center, III Michigan \\ Avenue, NW, Washington DC 200 I0, USA; ${ }^{3}$ Department of Pediatrics, George Washington University School of Medicine and Health Sciences, \\ Washington DC 200 I0, USA
}

Recent findings link increased expression of the structurally complex 'b' pathway gangliosides GDIb, GTIb, GQIb (CbG) to a favourable clinical and biological behaviour in human neuroblastoma (NB). Seeking a model to probe these observations, we evaluated four human NB cell lines. Very low CbG content (4-10\%) in three of the four cell lines (LAN-5, LAN-I, SMS-KCNR) reflected the ganglioside pattern observed in the most aggressive NB tumours. Pharmacological alterations of complex ganglioside synthesis in vitro by a 5-7 day exposure to 5-10 $\mu \mathrm{M}$ retinoic acid, which is employed in maintenance therapy of disseminated NB, included markedly increased (i) relative expression of $\mathrm{CbG}(6.6 \pm 2.0$-fold increase, $P=0.037$ ), (ii) relative expression of the analogous 'a' pathway gangliosides, termed $\mathrm{CaG}(6.4 \pm 1.4$-fold increase in GMIa and GDIa; $P=0.010)$, and (iii) total cellular ganglioside content (2.0-6.3-fold), which in turn amplified the accumulation of structurally complex gangliosides. Substantial increases $(2.7-2.9$-fold $)$ in the activity of GDIb/GMla synthase ( $\beta$-1,3-galactosyltransferase), which initiates the synthesis of $\mathrm{CbG}$ and $\mathrm{CaG}$, accompanied the all-trans retinoic acid (ATRA)-induced ganglioside changes. Thus, increased CbG synthesis in NB cell lines is attributable to a specific effect of ATRA, namely induction of GD I b/GMI a synthase activity. Since the shift towards higher expression of $\mathrm{CbG}$ and $\mathrm{CaG}$ during retinoic acid-induced cellular differentiation reflects a ganglioside pattern found in clinically less-aggressive tumours, our studies suggest that complex gangliosides may play a role in the biological and clinical behaviour of NB.

British Journal of Cancer (2004) 91, 389-397. doi:I0.1038/sj.bjc.6601914 www.bjcancer.com

Published online 8 June 2004

(c) 2004 Cancer Research UK

Keywords: neuroblastoma; gangliosides; retinoic acid; GDIb synthase

Increasing evidence has implicated gangliosides, a specific class of cell surface glycosphingolipids, in the biological and clinical behaviour of many types of tumours including human neuroblastoma (NB). Gangliosides are overexpressed and actively shed by tumour cells (Wu et al, 1986; Valentino et al, 1990; Li and Ladisch, 1991) and have a number of biological properties that could conceivably alter tumour-host interactions to influence the survival of the malignant cells that carry these molecules (Hakomori, 1996). Our previous studies have linked specific ganglioside changes in human NB tumours to differences in the clinical and biological behaviour of this tumour (Kaucic et al, 2001; Hettmer et al, 2003), and raise the possibility that pharmacologically induced modulation of NB ganglioside content could have important consequences for outcome.

Gangliosides consist of a sialic acid-containing carbohydrate portion and a lipid portion (ceramide) embedded in the outer leaflet of the cell membrane. They are synthesised via two prominent pathways, designated 'a' (GM2, GM1a, GD1a) and 'b' (GD3, GD2, GD1b GT1b, GQ1b), from a common precursor (GM3) derived from lactosylceramide (Figure 1). Each ganglioside is structurally more complex than

\footnotetext{
* Correspondence: Dr K Kaucic, Center for Cancer and Immunology Research, Children's National Medical Center, II I Michigan Avenue NW, Washington DC 200 I0, USA; E-mail: kkaucic@cnmc.org Received 10 February 2004; revised 21 April 2004; accepted 23 April 2004; published online 8 June 2004
}

its precursor molecule, and the stepwise addition of monosaccharide or sialic acid residues by specific membrane-bound glycosyltransferases in the Golgi apparatus is catalysed by the same glycosyltransferases in both pathways (van Echten and Sandhoff, 1993).

Our previous studies have characterised ganglioside expression in human NB, a paediatric malignancy of neural crest origin (Wu et al, 1986; Valentino et al, 1990; Kaucic et al, 2001; Hettmer et al, 2003). We have linked alterations in the expression of complex ' $b$ ' pathway gangliosides (CbG) downstream of GD1b/GM1a synthase (GD1b, GT1b and GQ1b) to differences in the biological phenotype and clinical behaviour that can be used to predict patient outcome in NB (Hettmer et al, 2003). Further, recent experimental evidence suggests that GD1b, GT1b and GQ1b can modulate a number of biological processes that are believed to counteract malignant transformation and progression, including tumour cell proliferation, host immune function and signal transduction mechanisms (Hynds et al, 1995; Fukumoto et al, 2000; Kanda et al, 2001; Kanda and Watanabe, 2001). We hypothesise that high CbG content may contribute to reduced tumour aggressiveness, and that alteration of CbG expression might be a potential therapeutic target in NB.

One pharmacological agent that is well known to alter cellular ganglioside metabolism and successfully used in the oral maintenance therapy of disseminated NB is retinoic acid (Matthay et al, 1999). Previous in vitro studies have shown that morphological signs of neuronal differentiation in response to retinoic acid are accompanied by an increase in total ganglioside content and a 


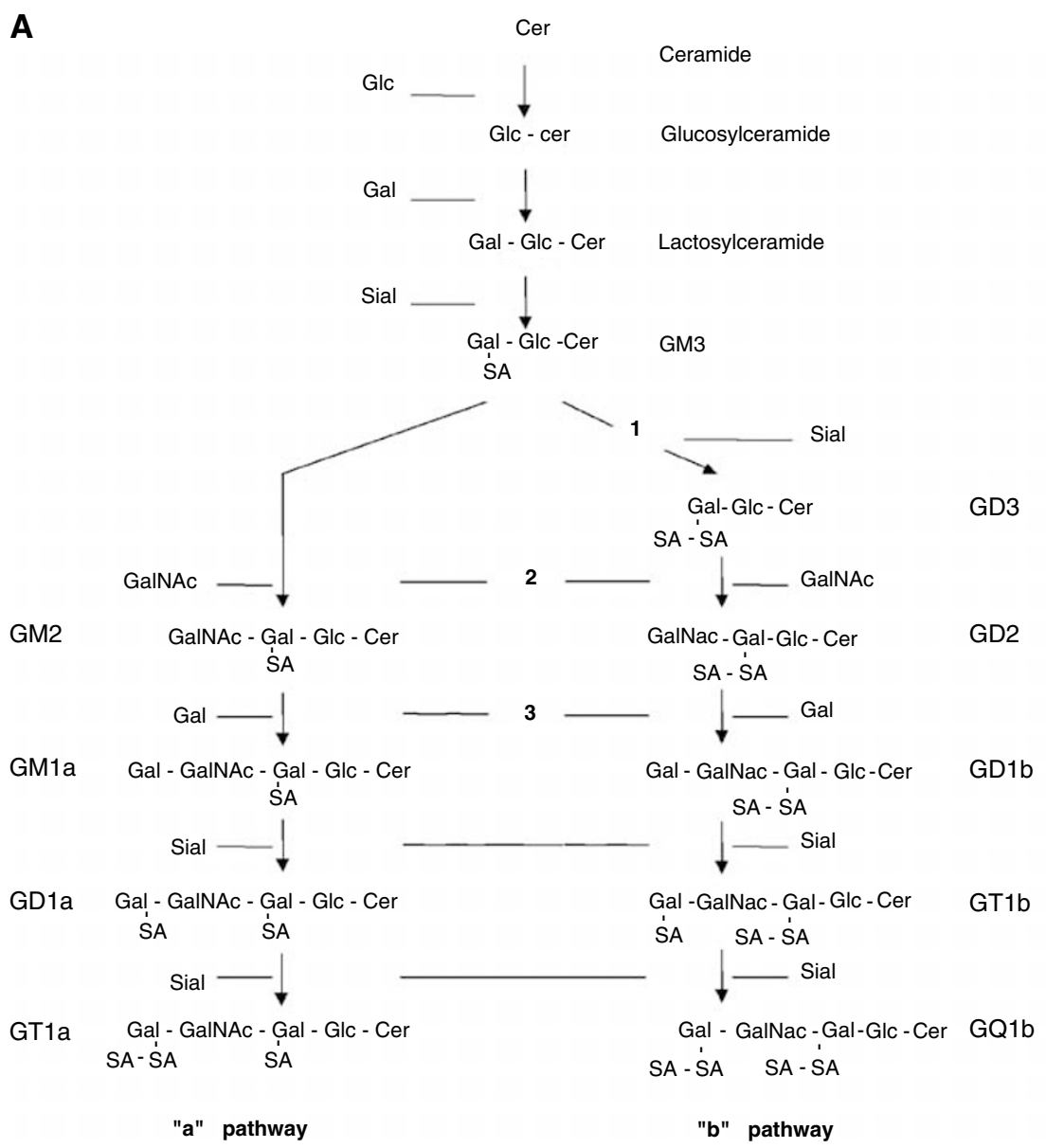

B

Glycosyltransferases Accession numbers NCBI Protein Bank

GD3 synthase

$\alpha$-2,8-sialyltransferase

AAA62366

SAT-II, ST8Sia

2

GD2 / GM2 synthase

$\beta$-1,4- $N$-acetylgalactosaminyltransferase

AAA35516

GD1b / GM1a synthase

$\beta$-1,3-galactosyltransferase

BAA88988

Figure I Schematic representation of the major pathways of ganglioside biosynthesis. (A) The monosialoganglioside GM3, derived from lactosylceramide, is the common precursor for both 'a' and 'b' pathway gangliosides. Each ganglioside species consists of a ceramide backbone (CER), and a carbohydrate chain $(g \mid c=$ glucose, gal = galactose, GalNAc =N-acetylgalactosamine) containing one or more sialic acid (SA) residues. 'a' and 'b' pathway gangliosides downstream of GDIb/GMla synthase were designated complex 'a' $(\mathrm{CaG})$ and complex 'b' $(\mathrm{CbG})$ gangliosides, respectively. (B) Parallel steps in both pathways are catalysed by the same glycosyltransferases of the Golgi apparatus (Li and Ladisch, 1997): (1), GD3 synthase ( $\alpha$-2,8-sialyltransferase): (2), GM2/ GD2 synthase ( $\beta$-I,4-N-acetylgalactosaminyltransferase); (3), GDIb/GMla synthase ( $\beta$-I,3-galactosyltransferase); (4), GTIb/GDIa synthase ( $\alpha$-2,3sialyltransferase); (5), GQ I b/GT la synthase ( $\alpha$-2,8-sialyltransferase). For each enzyme, accession numbers according to the NCBI protein bank are included.

relative increase in the expression of certain complex gangliosides of both the ' $a$ ' and ' $b$ ' pathway in NB cells (Li and Ladisch, 1992; Rebhan et al, 1994), as well as in normal and transformed embryonal cells (Levine and Flynn, 1986; Rizzo et al, 1995; Liour et al, 2000). On the basis of these findings, we have undertaken a comprehensive analysis of retinoic acid-induced changes in ganglioside expression and metabolism in human NB cell lines.

Here, we demonstrate that low CbG levels in some NB cell lines are analogous to the ganglioside pattern observed in clinically and biologically unfavourable NB tumours, providing an in vitro model to study pharmacological agents that might alter the metabolism of complex ganglioside subspecies. We further demonstrate that treatment with retinoic acid markedly enhances the activity of GD1b/GM1a synthase, resulting in increased expression of the complex gangliosides downstream of this enzyme, namely GD1b and GT1b (CbG), and GM1a and GD1a (CaG). Our results thus identify a specific effect of retinoic acid on the expression and biosynthesis of complex gangliosides in NB cells. 


\section{MATERIALS AND METHODS}

\section{Cell culture}

Human NB cell lines LAN-1, LAN-5, SMS-KCN and SMS-KCNR were derived from three children with disseminated NB and have been previously described. SMS-KCN and SMS-KCNR were established from the same patient, from the primary tumour and bone marrow at relapse, respectively (Kohl et al, 1983; Reynolds et al, 1986; Rettig et al, 1987).

LAN-1 and LAN-5 were maintained in Waymouth's MB 752/1 medium (GIBCO, Grand Island, NY, USA), and SMS-KCN and SMS-KCNR in RPMI 1640 medium (GIBCO, Grand Island, NY, USA). The medium was supplemented with $2 \mathrm{~mm}$ L-glutamine and $10 \%$ heat-inactivated foetal calf serum (GIBCO, Grand Island, NY, USA), respectively. Cells were grown in adherent monolayer cultures using $75-\mathrm{cm}^{2}$ and $175-\mathrm{cm}^{2}$ cell culture flasks (Becton Dickinson, Franklin Lakes, NJ, USA) at $37^{\circ} \mathrm{C}$ in a $5 \% \mathrm{CO}_{2}$ atmosphere in a humidified incubator

Retinoic acid (all-trans or 13-cis; Sigma Chemical Co., St Louis, MO, USA) was dissolved in ethanol to a concentration of $10^{-2} \mathrm{M}$ and kept as stock solution for up to 4 weeks at $-70^{\circ} \mathrm{C}$, protected from light. For each experiment, stock solutions were diluted with the growth medium to a final concentration of 5 or $10 \mu \mathrm{M}$ retinoic acid. These concentrations are equivalent to the serum levels effective in the oral maintenance therapy of disseminated NB with 13-cis retinoic acid (13-cis RA) $(7.2 \pm 5.3 \mu \mathrm{M})$ (Reynolds and Lemons, 2001). The final concentration of ethanol in the culture medium was $\leqslant 0.2 \%\left(\mathrm{vv}^{-1}\right)$.

Cells were seeded at $7 \times 10^{4}$ cells $\mathrm{cm}^{-2}$. After $24 \mathrm{~h}$, the medium was replaced with a medium containing retinoic acid. Culture medium was replaced with fresh medium containing retinoic acid every other day. Untreated control cells were grown with and without ethanol. Cells were harvested after 5-7 days of exposure to retinoic acid, followed by ganglioside purification or extraction of crude membrane extracts, as described below. Viable cells were counted using trypan blue dye exclusion and the cellular protein content was estimated by the Bio-Rad $\mathrm{D}_{\mathrm{C}}$ Protein Assay (Bio-Rad Laboratories, Hercules, CA, USA), using a bovine serum albumin standard.

\section{Ganglioside purification and quantification}

Methods for extraction and purification of gangliosides were previously described (Ladisch and Gillard, 1985). Briefly, total lipid extracts of cell pellets were obtained by extracting the lyophilised cell pellets twice with chloroform/methanol $\left(1: 1 \mathrm{vv}^{-1}\right)$ at $4{ }^{\circ} \mathrm{C}$ with stirring. Gangliosides were isolated by partitioning the dried total lipid extract in diisopropyl ether/1-butanol/17 mM aquaeous $\mathrm{NaCl}$ $\left(6: 4: 5 \mathrm{vv}^{-1}\right)$. Gangliosides, in the lower aqueous phase, were further purified by Sephadex G-50 gel filtration.

High-performance thin layer chromatography (HPTLC) analysis of gangliosides (Ledeen and $\mathrm{Yu}, 1982$ ) was performed using $10 \times 10$ - or $10 \times 20 \mathrm{~cm}$ precoated silica gel HPTLC plates (Merck, Darmstadt, Germany). The plates were developed in chloroform/ methanol/0.25\% $\mathrm{CaCl}_{2} \cdot \mathrm{H}_{2} \mathrm{O}$ (60:40:9 by volume), and the gangliosides were stained with resorcinol. Absolute cellular ganglioside content was determined by HPTLC densitometry using known concentrations of human brain gangliosides (HBG) as the standard. Radioactive products were visualised by exposure of HPTLC plates to an X-ray film (BioMax, Kodak).

Individual gangliosides were identified using a human brain ganglioside standard or ${ }^{14} \mathrm{C}$-labelled rat brain gangliosides. The area under the peak generated by each ganglioside band was integrated after the plates/films were scanned (Microtek ScanMaker 5), and the relative percentage was calculated. To categorise individual ganglioside species, we used a previously established classification scheme based on biosynthetic pathways (Hettmer et al, 2003). Structurally complex molecules downstream of GD1b/ GM1a synthase ( $\beta$-1,3-galactosyltransferase) were designated complex 'a' gangliosides (CaG: GM1a, GDla and GT1a) and complex ' $b$ ' gangliosides (CbG: GD1b, GT1b and GQ1b), respectively.

\section{Glycosyltransferase assays}

The enzymatic activities of $\alpha$-2,8-sialyltransferase (GD3 synthase), $\beta$-1,4- $N$-acetylgalactosaminyltransferase (GD2 synthase) and $\beta$-1,3glactosyltransferase (GD1b synthase) were measured in membrane extracts from retinoic acid-treated and control cells by incorporation of radiolabelled sugars into ganglioside precursors (Kemp and Stoolmiller, 1976; Pohlentz et al, 2000).

Preparation of crude membrane extracts Crude membrane fractions, used as the source of enzymes, were prepared by suspending cell pellets in five volumes of cold $0.25 \mathrm{M}$ sucrose containing $0.01 \mathrm{~m}$ 2-mercaptoethanol. Cells were disrupted using eight cycles of freezing in liquid nitrogen and thawing in a water bath at $37^{\circ} \mathrm{C}$. After centrifugation at $2000 \mathrm{~g}$ for $10 \mathrm{~min}$ to remove cellular debris, the supernatant fractions were centrifuged at $10000 \mathrm{~g}$ for $10 \mathrm{~min}$ and then sedimented at $100000 \mathrm{~g}$ for $1 \mathrm{~h}$ (Kemp and Stoolmiller, 1976). The sedimentable fractions were resuspended in unbuffered sucrose $(0.25 \mathrm{M})$ and the amount of membrane protein was determined using the Bio-Rad $D_{C}$ Protein Assay (Bio-Rad Laboratories, Hercules, CA, USA).

Processing of glycosyltransferse assays Triton X-100 (50 $\mu \mathrm{g}$, Sigma Chemical Co., St Louis, MO, USA), ganglioside precursors and radiolabelled sugar donors in chloroform/methanol $\left(1: 1 \mathrm{vv}^{-1}\right)$ were transferred to $1 \mathrm{ml}$ conical glass tubes and the solvent was evaporated under a stream of nitrogen. The corresponding buffer mixture and unlabelled sugar donor were added, the mixture was shaken vigorously, and each sample was sonicated for $30 \mathrm{~s}$. Reaction mixtures were completed by addition of $50-150 \mu \mathrm{g}$ membrane protein to a final reactive volume of $50 \mu \mathrm{l}$ and shaken gently. After incubation at $37^{\circ} \mathrm{C}$ for $1 \mathrm{~h}$, reactions were terminated by addition of $1 \mathrm{ml}$ chloroform/methanol $\left(2: 1 \mathrm{vv}^{-1}\right)$ (Kemp and Stoolmiller, 1976; Pohlentz et al, 2000).

$\alpha$-2,8-sialyltransferase (GD3 synthase) activity was determined using $30 \mathrm{nmol}$ GM3 (Sigma Chemical Co., St Louis, MO, USA), $0.01 \mu \mathrm{Ci}{ }^{14} \mathrm{C}$-labelled CMP sialic acid ([sialic-4,5,6,7,8,9- ${ }^{14} \mathrm{C}$, $150 \mathrm{mCi} \mathrm{mmol}^{-1}$ ), $10 \mathrm{nmol}$ 'cold' CMP sialic acid, $2.5 \mu \mathrm{mol}$ of MES buffer ( $\mathrm{pH} 5.9$ ), $2.5 \mu \mathrm{mol}$ of $\mathrm{KCl}, 0.1 \mu \mathrm{mol}$ of $\mathrm{MnCl}_{2}$ and $0.3 \mu \mathrm{mol}$ of $\mathrm{MgCl}_{2}$.

To assess $\beta$-1,4-N-acetylgalactosaminyltransferase (GD2 synthase) activity, $30 \mathrm{nmol}$ GD3 (Matreya Inc., Pleasant Gap, PA, USA), $0.01 \mu \mathrm{Ci}{ }^{3} \mathrm{H}$ ]-labelled UDP $N$-acetyl-D-galactosamine ([galactosamine- $\left.\left.1-{ }^{3} \mathrm{H}(N)\right], 5 \mathrm{Ci} \mathrm{mmol}^{-1}\right), 14 \mathrm{nmol}$ 'cold' $N$-acetyl-Dgalactosamine, $2.5 \mu \mathrm{mol}$ MES ( $\mathrm{pH}$ 6.5) and $1.5 \mu \mathrm{mol} \mathrm{MnCl}_{2}$ were used.

For evaluation of $\beta$-1,3-glactosyltransferase (GD1b synthase) activity, the reaction mixture contained $10 \mathrm{nmol}$ GD2 (Sigma Chemical Co., St Louis, MO, USA), $0.01 \mu \mathrm{Ci}{ }^{14} \mathrm{C}$-labelled UDP galactose ([galactose $\left.\left.-{ }^{14} \mathrm{C}\right](\mathrm{U}), 250 \mathrm{mCi} \mathrm{mmol}^{-1}\right), 20 \mathrm{nmol}$ 'cold' galactose, $2.5 \mu \mathrm{mol} \mathrm{MES} \mathrm{(pH} \mathrm{6.5)} \mathrm{and} 2.1 \mu \mathrm{mol} \mathrm{MnCl}_{2}$.

For each glycosyltransferase assay, buffer mixtures containing MES (Calbiochem, San Diego, CA, USA), $\mathrm{KCl}, \mathrm{MnCl}_{2}$ and $\mathrm{MgCl}_{2}$ (Sigma Chemical Co., St Louis, MO, USA) were freshly prepared. Radiolabelled and 'cold' sugar donors were obtained from PerkinElmer Life Sciences Inc. (Boston, MA, USA) and Calbiochem (San Diego, CA, USA), respectively. All enzyme assays were performed in duplicates with the appropriate blank.

Separation of lipid-soluble reaction products Lipid-soluble compounds in the reaction mixture were isolated by passing them through columns of Sephadex G25 Superfine $(1 \times 3-5 \mathrm{~cm}$ in 
program. Enzyme activity $\left(\mathrm{pmol} \mathrm{h}^{-1} \mathrm{mg}_{\text {protein }}{ }^{-1}\right.$ ) was calculated from the ratio of the products and the radioactivity of the total sample. The remainder of the sample was used to confirm the identity and the purity of the product by TLC autoradiography.

\section{Statistical analysis}

The relative expression of individual gangliosides and ganglioside subsets $(\mathrm{CbG}$ and $\mathrm{CaG})$ in each of the four cell lines studied were expressed as the mean \pm s.e.m. for three to five separate experiments. For the three cell lines that were responsive to retinoic acid, the relative ganglioside content was similarly reported as the mean \pm s.e.m. and, alternatively, the per cent of the expression of parallel control cells for two separate experiments per cell line. Data from six separate experiments involving three different cell lines were normalised by relating results in each experimental group to their respective controls by expressing the experimental (exp) levels as a per cent of the control (ctrl) levels $(\exp / \mathrm{ctrl} \times 100)$. Control values of zero were set to 1 before the computation. To test the hypothesis that experimental values differed from controls, we employed a $t$-test to examine whether the experimental values differed from $100 \%$. The same method was used to compare glycosyltransferase activities among retinoic acid-treated and control cells in retinoic acid-responsive cell lines (LAN-1, LAN-5, SMS-KCNR). Results were considered statistically significant if the $P$-value was less than 0.050 .

To examine whether experimental values differed from controls in studies of 13-cis RA, mean treatment values were compared to control values (no treatment with 13 -cis RA) using a $t$-test.

\section{RESULTS}

\section{Ganglioside expression in human NB cell lines}

The impetus for this study were our findings (Hettmer et al, 2003) and those of others (Schengrund et al, 1985; Sung et al, 1995; Yates et al, 1999), suggesting that differences in tumour tissue CbG (GD1b, GT1b and GQ1b) expression are linked to the clinical and biological behaviour of NB and other tumours. In order to obtain an in vitro model that permits a more dynamic view of $\mathrm{NB}$ ganglioside biosynthesis, we analysed ganglioside expression in four well-described NB cell lines, LAN-1, LAN-5, SMS-KCNR and SMS-KCN.

To analyse ganglioside expression, we grew NB cells to $80-90 \%$ confluence, then extracted total cellular gangliosides and separated them by HPTLC (Figure 2). Gangliosides were measured (i) as absolute cellular ganglioside content (nmol lipid-bound sialic acid (LBSA) $10^{8}$ cells $^{-1}$ ) and (ii) as relative percentages of individual gangliosides out of total cellular gangliosides. Absolute cellular ganglioside content differed widely among the NB cell lines studied (69 nmol LBSA $10^{8}$ cells $^{-1}$ for SMS-KCN, $57 \mathrm{nmol} \mathrm{LBSA} 10^{8} \mathrm{cells}^{-1}$ for LAN-5, $30 \mathrm{nmol}$ LBSA $10^{8}$ cells $^{-1}$ for LAN-1 and $15 \mathrm{nmol}$ LBSA $10^{8}$ cells $^{-1}$ for SMS-KCNR). Nevertheless, there were clear similarities in the relative ganglioside content of the four cell lines (Table 1). The most prominent components were the ' $b$ ' pathway disialoganglioside GD2 (26-60\%) and the 'a' pathway monosialoganglioside GM2 (22-35\%). Complex ' $b$ ' pathway gangliosides represented only minor components $(\leqslant 10 \%)$ in three of the four NB cell lines studied ( $10 \%$ in LAN-5, $4 \%$ in LAN-1 and $8 \%$ in SMS-KCNR), and were composed of the disialylated molecule GD1b (2\%) and the trisialylated ganglioside GT1b $(2-8 \%)$. The fourth cell line, SMS-KCN, contained $21 \%$ CbG (2\% GD1b and 19\% GT1b). There were no detectable amounts of the most complex molecule, GQ1b, in any of the cell lines studied.

The prominent position of GD2 as a major ganglioside component in the NB cell lines studied (44\% in LAN-5, 36\% in LAN-1, $60 \%$ in SMS-KCNR and $26 \%$ in SMS-KCN) mirrors the 
Table 2 Effect of ATRA on neuroblastoma cell ganglioside expression

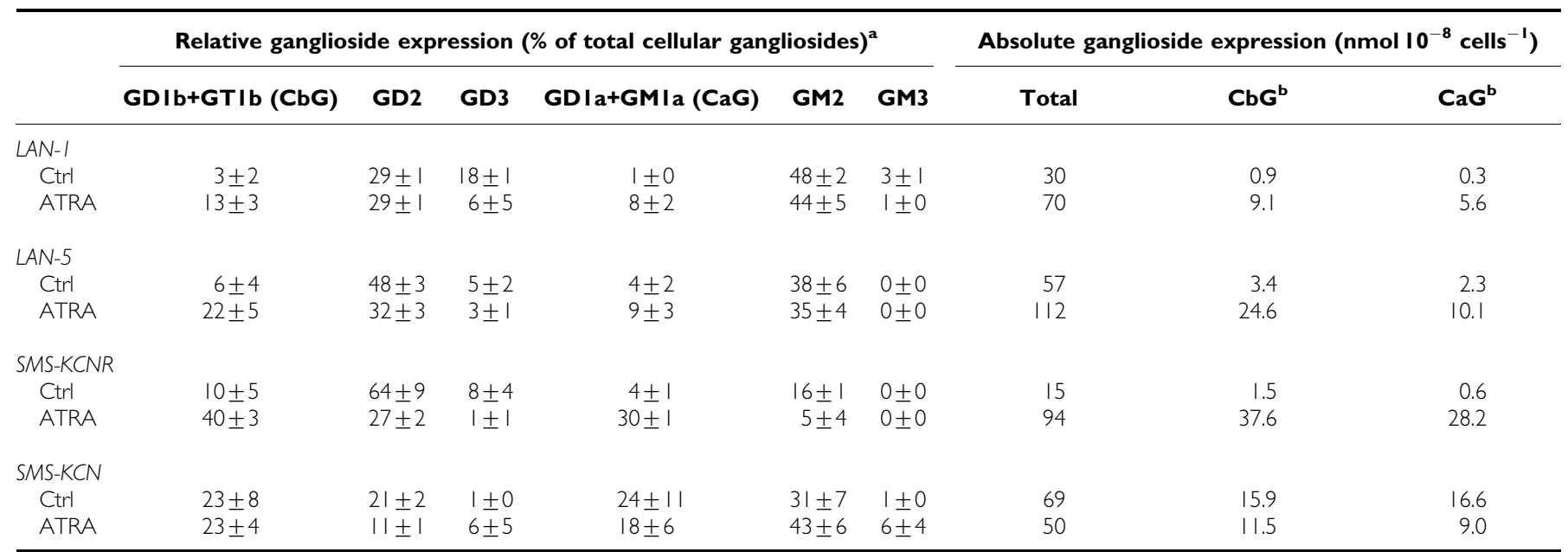

${ }^{a} V$ alues represent means \pm standard errors of two independent experiments. ${ }^{b}$ Calculated from the absolute total ganglioside content and the mean relative content of CaG or $\mathrm{CbG}$. ATRA = all-trans retinoic acid; Ctrl = control; $\mathrm{CbG}=$ complex 'b' pathway gangliosides; $\mathrm{CaG}=$ complex 'a' pathway gangliosides.

Table 3 Relative changes in neuroblastoma cell ganglioside expression in ATRA-responsive cell lines

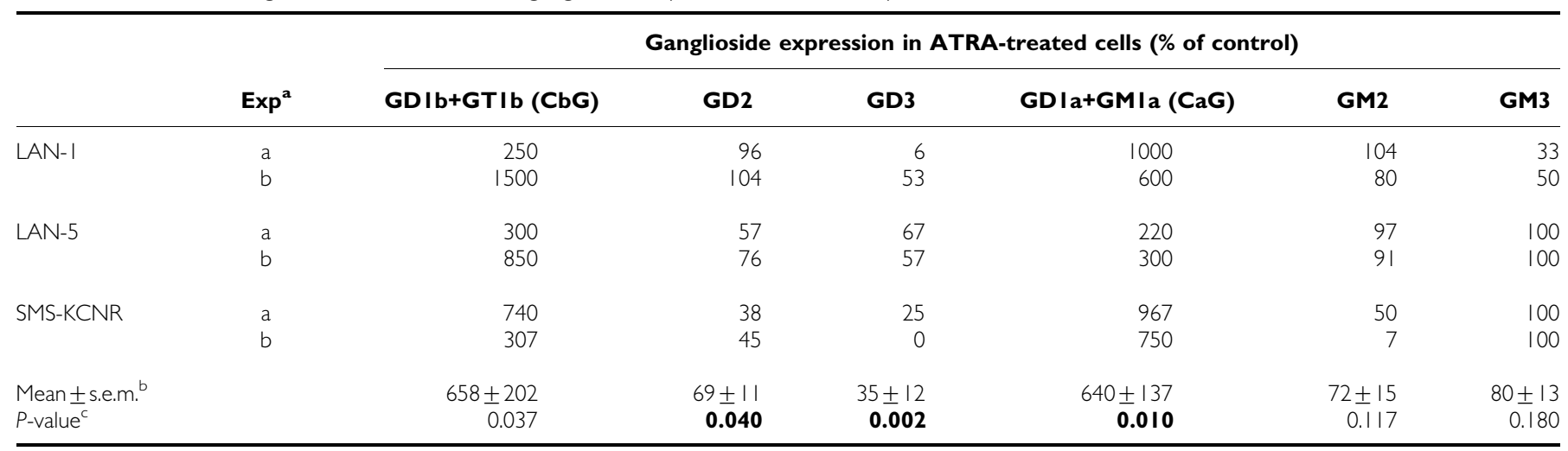

${ }^{a}$ For each cell line, two separate experiments ( $a$ and $b$ ) were analysed. ' $M e a n \pm$ s.e.m. of normalised values for all three cell lines (two experiments per cell line; six experiments total). Bold values are statistically significant $(P<0.050)$. ' $t$-tests. ATRA = all-trans retinoic acid; $C b G=$ complex 'b' pathway gangliosides; $C a G=$ complex 'a' pathway gangliosides.

ubiquitously high expression of GD2 that characterizes $\mathrm{NB}$ tumours ( $\mathrm{Wu}$ et al, 1986). Importantly, the low CbG expression observed in each of the cell lines is also analogous to the CbG levels found in clinically and biologically unfavourable NB tumours (Hettmer et al, 2003). We therefore used these four NB cell lines as an in vitro model to determine whether retinoic acid, as a pharmacological agent, might alter the expression and biosynthesis of CbG.

\section{Retinoic-acid induced changes in CbG expression in NB cell lines}

Retinoids are a class of vitamin A derivatives that differ in their isomeric conformations, and include all-trans retinoic acid (ATRA), 9-cis retinoic acid and 13-cis RA. Owing to its superior pharmacokinetics (higher and more sustained plasma levels), 13cis RA is the isomer that is primarily used clinically. However, 13cis RA isomerises in vivo to both ATRA and 9-cis retinoic acid, which are believed to be the active compounds in vivo in nonocular tissue (Lovat et al, 1994). Since retinoic acid treatment has been associated with changes in the ganglioside complement of various cell types (Li and Ladisch, 1992; Rebhan et al, 1994; Liour et al, 2000), and the oral maintenance therapy of disseminated NB with 13-cis RA results in improved event-free survival (Matthay et al, 1999), we sought to specifically explore the effect of retinoic acid on CbG expression. We, therefore, first treated NB cells in vitro with $10 \mu \mathrm{m}$ ATRA, then evaluated ATRA-induced changes in the composition of individual gangliosides and in total cellular ganglioside content in LAN-1, LAN-5, SMS-KCNR and SMS-KCN cells (Table 2). Strikingly, ATRA induced a shift from synthesis of simpler gangliosides towards more complex species within both the ' $a$ ' and ' $b$ ' pathways in three of the four cell lines (LAN-1, LAN5 and SMS-KCNR). In these three cell lines, the redistribution towards complex gangliosides included increased relative expression of GD1b and GT1b ('b' pathway) and GM1a and GD1a ('a' pathway), compounded by a substantial increase in total cellular ganglioside content, making the increase in the absolute content of $\mathrm{CbG}$ and $\mathrm{CaG}$ even more striking (Table 2). In LAN-5 cells for example, the ATRA-induced changes in cellular ganglioside expression included a 3.7-fold increase in $\mathrm{CbG}$ content (from 6 to 22\%), a 2.2-fold increase in $\mathrm{CaG}$ content (from 4 to $9 \%$ ) and a two-fold increase in absolute ganglioside content (from 57 to $112 \mathrm{nmol} 10^{8}$ cells $^{-1}$ ). SMS-KCN, the cell line with the highest baseline level of $\mathrm{CbG}$, was the only cell line of the four which was not responsive to ATRA treatment; specifically, the change in complex ganglioside content was minimal (23 vs $23 \% \mathrm{CbG}$ and 24 vs $18 \% \mathrm{CaG}$ in controls compared to ATRAtreated cells), as was the change in total cellular ganglioside 
A

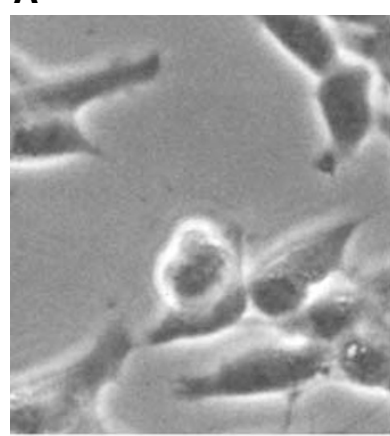

Ctrl

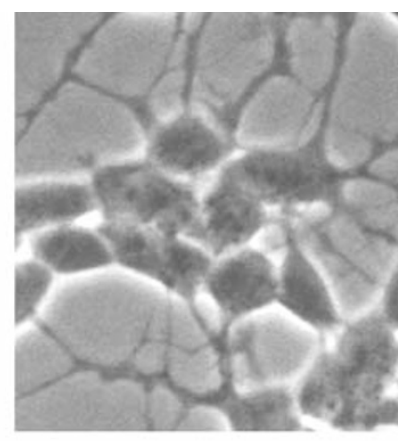

$10 \mu \mathrm{M}$ ATRA

B

B

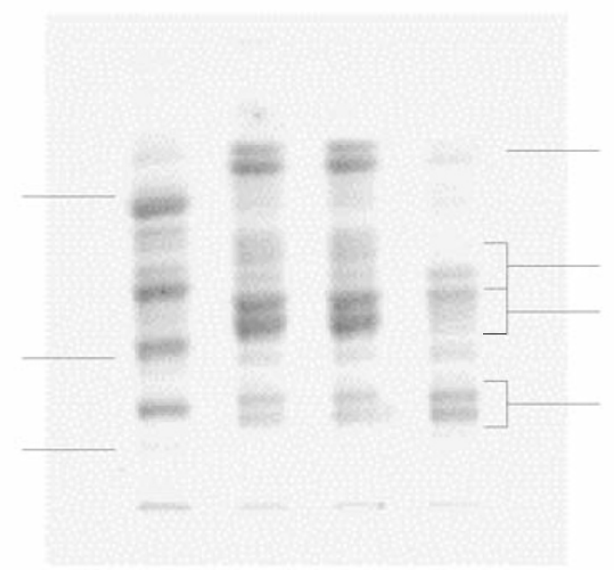

GM2

GM1a

HBG Ctrl Ctrl* ATRA

Figure 3 Effect of all-trans retinoic acid (ATRA) on cell morphology $(\mathbf{A})$ and cellular ganglioside expression (B) in the human NB cell line SMSKCNR. Cells were plated at $7 \times 10^{4}$ cells and treated with ATRA $10 \mu \mathrm{M}$ on days $1-7$. Photomicrograph $(\times 100)$ was obtained on day 6 . Cells were harvested on day 7 , and gangliosides were purified and separated by HPTLC (Ctrl, medium alone; Ctrl*, medium containing 0.1\% ethanol; ATRA, medium containing $10 \mu \mathrm{M}$ ATRA). GD2, GDIa and GTIb are doublets in tumour samples. The lower GDIa band and the upper GD2 band overlap.

Table 4 Effect of ATRA on neuroblastoma (NB) cell glycosyltransferase activities

\begin{tabular}{lccc}
\hline & \multicolumn{2}{c}{ Enzyme activity $\left(\mathbf{p m o l} \mathbf{~}^{-1} \times \mathbf{~ m g ~ p r o t e i n ) ~}^{\mathbf{a}}\right.$} \\
\cline { 2 - 4 } & GD Ib synthase & GD2 synthase & GD3 synthase \\
\hline LAN-5 & & & \\
Ctrl & 215 & 1416 & 572 \\
ATRA & 572 & 1384 & 669 \\
SMS-KCNR & & & \\
Ctrl & 172 & 518 & 364 \\
ATRA & 497 & 927 & 511 \\
SMS-KCN & & & \\
Ctrl & 186 & 1143 & 333 \\
ATRA & 283 & 769 & 405 \\
\hline
\end{tabular}

${ }^{a}$ Activities of GD I b synthase, GD2 synthase and GD3 synthase in NB cells treated with $10 \mu \mathrm{M}$ all-trans retinoic acid (ATRA) are compared to untreated cells (Ctrl). Enzyme activities were determined by incorporation of radiolabelled sugars into ganglioside precursors. Values represent means from duplicate samples of one to two experiments. ATRA $=$ all-trans retinoic acid; $C$ trl $=$ control; $C b G=$ complex ' $b$ ' pathway gangliosides; $\mathrm{CaG}=$ complex 'a' pathway gangliosides.

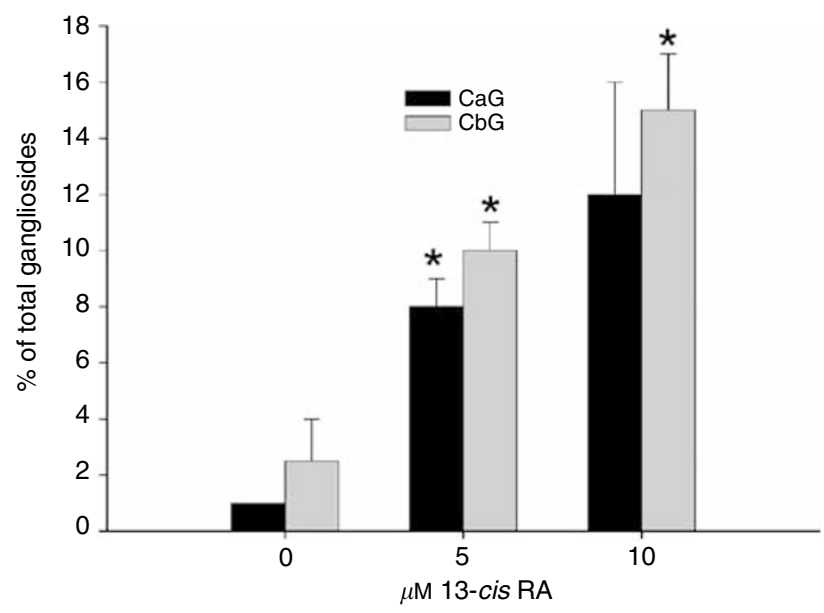

Figure 4 Effect of I3-cis retinoic acid (I3-cis RA) on CbG (GDIb, GTIb; black bars) and CaG (GMIa, GDIa; grey bars) expression in LAN-I human NB cells. Bars represent means \pm s.e.m. Ganglioside subsets are expressed as a percentage of total cellular gangliosides. Experimental and controls values were compared using a $t$-test $(* P<0.050)$.

expression (69 vs $50 \mathrm{nmol} 10^{8}$ cells $^{-1}$ ). When normalised data from the three responsive cell lines were analysed together (Table 3), ATRA treatment clearly resulted in significant increases in the cellular content of CbG $(658 \pm 202 \%$ of control, $P=0.037)$ and $\mathrm{CaG}(640 \pm 137 \%$ of control, $P=0.010)$, and, concomitantly, significant decreases in the cellular content of GD2 $(69 \pm 11 \%$ of control; $P=0.040)$ and GD3 $(35 \pm 12 \%$ of control; $P=0.002$ )

To illustrate these changes, Figure 3 summarises the ATRAinduced changes in cell morphology and ganglioside expression of the NB cell line SMS-KCNR. The morphological changes in response to ATRA, including rounding up of the cell body, extension of long processes with the appearance of neurites and cell aggregation into tight clusters (Figure 3A), are consistent with previous studies evaluating the differentiating effects of ATRA on NB cells (Li and Ladisch, 1992; Melino et al, 1997). Qualitative changes in the composition of cell-surface gangliosides accompanied morphological signs of differentiation in response to ATRA. The most prominent ganglioside components in untreated SMS-KCNR cells were the ' $b$ ' pathway ganglioside GD2 and the ' $a$ ' pathway ganglioside GM2. Treatment with ATRA increased the proportions of the CbG (GD1b and GT1b), as well as the CaG (GD1a and GM1a), such that these two subgroups predominated relative to other ganglioside species (Figure 3B). In view of the increase in total cellular ganglioside content (Table 2), this relative change resulted in significant absolute changes in the membrane expression of complex ganglioside molecules. Thus, the mean four-fold increase in relative $\mathrm{CbG}$ expression translated into a 25 -fold increase in absolute cellular CbG expression in SMS-KCNR cells, indicating a striking accumulation of these biologically active molecules.

Since 13-cis RA is currently the only retinoid compound in clinical use to treat NB (Reynolds and Lemons, 2001), we also exposed one NB cell line, LAN-1, to 13-cis RA at two different concentrations $(5 \mu \mathrm{M}, 10 \mu \mathrm{M})$. We observed that 13-cis RA caused a concentration-related increase in the expression of structurally complex gangliosides in LAN-1 cells (Figure 4). As with ATRAtreated cells, the increase in CbG expression was paralleled by a concurrent increase in $\mathrm{CaG}$, suggesting a specific effect of retinoids on the activity of GD1b/GM1a synthase ( $\beta-1,3$-galactosyltransferase), which initiates the synthesis of both $\mathrm{CbG}$ and $\mathrm{CaG}$, from GD2 and GM2, respectively. 
Effect of retinoic acid on the activity of ganglioside glycosyltransferases in human NB cells in vitro

To delineate the metabolic basis for the ATRA-induced shift towards expression of complex gangliosides, we determined the effect of ATRA treatment on the activity of three key enzymes in the synthesis of these molecules. Precursor molecules enter ' $b$ ' pathway ganglioside biosynthesis through conversion of GM3 into GD3 by GD3 synthase ( $\alpha$-2,8-sialyltransferase). GD2/GM2 synthase $(\beta-1,4-N$-acetylgalactosaminyltransferase) and GD1b/GM1a synthase $(\beta$-1,3-glactosyltransferase) catalyse the two subsequent enzymatic reactions resulting in the synthesis of the most complex gangliosides in each pathway (van Echten and Sandhoff, 1993) (Figure 1). We measured the activities of GD3 synthase, GD2 synthase and GD1b synthase in crude membrane extracts from LAN-5, SMS-KCNR, SMS-KCN, using excess GM3, GD3 and GD2 as exogenously added ganglioside precursors.

When the retinoic acid-responsive LAN-5 or SMS-KCNR cells were exposed to $10 \mu \mathrm{M}$ ATRA for 6-7 days (Table 4), we observed a marked increase $(2.7-2.9$-fold, $P<0.050)$ in GD1b synthase activity. This was accompanied by a lesser (0-1.8-fold, $P=0.198)$ increase in GD2 synthase activity, and only small changes in GD3 synthase activity (1.2-1.4, $P=0.161)$. Thus, in both LAN-5 and SMS-KCNR cells, increased expression of complex gangliosides downstream of GD1b sythase was associated with a significant (over two-fold) increase in GD1b synthase activity. In contrast, in SMS-KCN cells, the cell line that was unresponsive to ATRA treatment, GD1b synthase activity was only slightly increased (1.5-fold) in ATRA-treated $v s$ control cells.

Figure 5 illustrates the autoradiographic visualisation of enzyme products resulting from ATRA-induced changes in ganglioside metabolism in SMS-KCNR cells, confirming the major increase in GD1b synthase activity (Figure 5A), a comparatively minor increase in GD2 synthase activity (Figure 5B) and almost no change in GD3 synthase activity (Figure 5C). It is interesting to note that in the case of the ATRA-treated cell samples, the parallel blank (which does not contain exogenously added ganglioside precursors) also shows the synthesis of GD1b and GM1a from the trace amounts of endogenous gangliosides present in the cell pellet (Figure 5A), further confirming the substantial increase in the activity of GD1b/GM1a synthase following treatment with ATRA. Taken together, our data demonstrate that the increase in GD1b synthase activity is specifically associated with increased expression of complex ganglioside species, induced by ATRA in NB cells in vitro, and provides strong evidence that elevated $\mathrm{CbG}$ and $\mathrm{CaG}$ expression can be accounted for by a specific effect of ATRA on this enzyme.

\section{DISCUSSION}

Treatment with retinoic acid is shown here to induce a dramatic shift from synthesis of simpler gangliosides towards predominant expression of structurally complex ' $a$ ' and ' $b$ ' pathway ganglioside molecules downstream of GD1b/GM1a synthase in some NB cell lines. The increase in total cellular ganglioside content combined with the relative changes in the proportion of complex gangliosides results in a significant increase in the absolute cellular content of these molecules. The findings are consistent with the previous observations of a dramatic increase in membrane expression and shedding of total cellular gangliosides and enhancement in GD1a and GT1b content in LAN-5 NB cells treated with retinoic acid ( $\mathrm{Li}$ and Ladisch, 1992).

Among the four cell lines employed in this study, the NB cell line SMS-KCN appears to be biologically distinct. Three differences are evident. First, SMS-KCN cells consist of two morphologically distinct cell populations, one neuroblastic (N-type) and the second substrate-adherent (S-type) with epithelial or fibroblast-like

\section{A GD1b synthase}

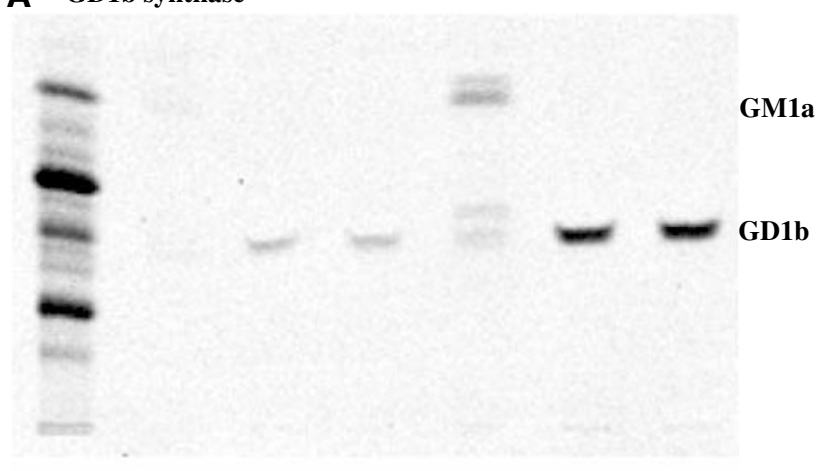

B GD2 synthase

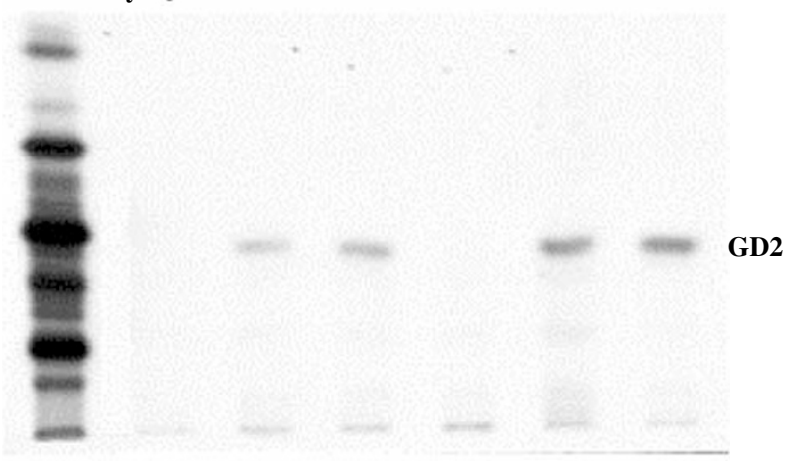

C GD3 synthase

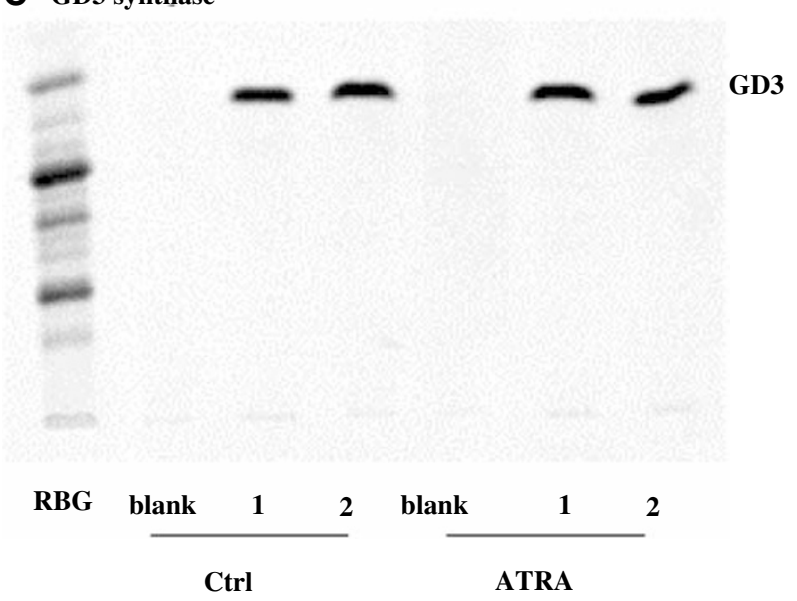

Figure 5 Effect of all-trans retinoic acid (ATRA) on the activities of GD I b synthase, GD2 synthase and GD3 synthase in the human NB cell line SMS-KCNR. HPTLC autoradiograms demonstrate reaction products from assays measuring activities of GDIb synthase $(\mathbf{A})$, GD2 synthase $(\mathbf{B})$ and GD3 synthase $(\mathbf{C})$. Enzyme assays were performed in duplicate $(1,2)$ with an appropriate 'blank' (Ctrl, medium alone; ATRA, $10 \mu \mathrm{M}$ ).

morphology (Rettig et al, 1987), while LAN-1, LAN-5 and SMSKCNR exhibit a uniform phenotype with small, round, neuroblastic cells that have short neuritic processes. Second, constitutive CbG content in SMS-KCN was higher (1.8- to 5.2-fold) than in the other cell lines studied. Third, treatment with retinoic acid did not induce the alterations in cellular ganglioside biosynthesis observed in the other cell lines. These differences in ganglioside metabolism 
do not have an obvious explanation, but it is possible that they are accounted for by constitutive differences between $\mathrm{N}$-type and S-type cells.

One possible explanation for the retinoic acid-induced changes in ganglioside composition in LAN-1, LAN-5 and SMS-KCNR cells is an increased rate of synthesis of certain gangliosides. A previous study in embryonal carcinoma cells demonstrated enhanced activities of various ganglioside glycosyltransferases at different stages of retinoic acid-induced neuronal differentiation, and a parallel increase in mRNA expression, suggesting that retinoic acid activated the transcription of these glycosyltransferases or stabilised their expression (Osanai et al, 1997; Liour et al, 2000). Our data support that the retinoic acid-induced increase in the expression of complex gangliosides both within the ' $a$ ' and the ' $b$ ' pathway is attributable to an induction of GD1b/GM1a synthase ( $\beta$-1,3-glactosyltransferase) activity. The retinoic acid-induced alterations in the ganglioside complement of NB cells seem to reflect, therefore, a specific effect on ganglioside anabolism, although, theoretically, alterations in ganglioside catabolism or shedding are also possible contributing factors. Increased expression of complex gangliosides is a well-described feature of normal embryonic neurogenesis and accelerated axonogenesis (Svennerholm et al, 1989; Hirschberg et al, 1996). Similar ganglioside changes to those we describe here have been reported in retinoic acid-treated Xenopus embryo cells, murine embryonal carcinoma cells and SHSY-5Y NB cells during neuronal maturation (Rebhan et al, 1994; Rizzo et al, 1995; Liour et al, 2000). Thus, predominant expression of complex gangliosides can be considered a biochemical marker of increasing neuronal differentiation. Further, the retinoic acid-induced changes in the ganglioside, complement of NB cell lines, are analogous to reported differences in $\mathrm{CbG}$ content among prognostically distinct categories of NB tumours (Schengrund et al, 1985; Schengrund and Shochat, 1988; Hettmer et al, 2003). Specifically, we previously found that among 74 human NB tumours, high $(\geqslant 35 \%)$ CbG expression (a) characterised nonprogressive $v s$ progressive tumours (median $41 v s$ 18\% of total gangliosides), and (b) was strongly predictive of a favourable outcome, even when other prognostic factors were considered (Hettmer et al, 2003). Related studies, associating low CbG levels in astrocytoma and medulloblastoma with higher histological grades and lower survival (Sung et al, 1995; Yates et al, 1999), further support the value of $\mathrm{CbG}$ in discriminating between different categories of malignant disease. In this context, the retinoic acid-induced increase in CbG expression in NB cells represents a transition into a ganglioside pattern which is associated with (i) neuronal differentiation in normal cells and (ii) clinically less-aggressive NB tumours, suggesting that CbGs may play a role in the biological behaviour of NB.

Tumour gangliosides of aberrant structure are generally believed to favour tumour progression (Hakomori, 1996), and a substantial body of previous experimental evidence associates the shedding of tumour cell surface gangliosides with increased tumorigenicity (Ladisch et al, 1987). The relationship between the concentration of shed GD2 in the plasma of NB-bearing patients and reduced long-term survival (Valentino et al, 1990) is consistent with this concept. There is also experimental evidence that suggests that the biological effects of tumour-derived gangliosides differ greatly depending on their carbohydrate and ceramide structures (Floutsis et al, 1989; Ladisch et al, 1994). Here we hypothesise that the significantly higher CbG levels in clinically favourable NB tumours (Hettmer et al, 2003) may lead to reduced NB tumour aggressiveness. Substantial experimental evidence corroborates the concept that the prognostic value of $\mathrm{CbG}$ reflects their functional involvement in the cellular processes that define the malignant behaviour of NB. Malignant transformation of murine epidermal cells in response to tumour-promoting phorbol esters is accompanied by a decrease in GT1b synthesis
(Srinivas and Colburn, 1982). Proliferation of and IL-8 production by human metastatic melanoma cells is inhibited by GD1b, GT1b and GQ1b (Kanda et al, 2001). Platelet-derived growth factor (PDGF)-mediated cell growth and PDGF receptor activation in $\mathrm{NB}$ and glioma cells are inhibited by complex ganglioside species (Hynds et al, 1995). These gangliosides also modulate immunoglobulin synthesis in peripheral blood mononuclear cells (Kanda and Tamaki, 1998, 1999a, b) and cause a shift from Th-2 to Th-1 cytokine production in PHA-stimulated $\mathrm{T}$ cells (Kanda et al, 2001). Finally, increased expression of GD1b and GT1b in rat pheochromocytoma cells transfected with GD3 synthase occurs in parallel with trk-A dimerisation (Fukumoto et al, 2000), which in turn is associated with improved prognosis in NB (Nakagawara et al, 1993). Taking these findings together, we hypothesise that CbG may impede tumour progression, either by acting directly on the tumour cell or by increasing the host resistance.

Similarly, increased tumour CbG expression might contribute to the success of retinoic acid in the maintenance therapy of disseminated NB. The beneficial role of retinoic acid is generally attributed to a reversal of the malignant state through differentiation of the tumour cells into more mature ganglion-like cells (Matthay et al, 1999; Reynolds and Lemons, 2001). Substantial experimental evidence suggests that complex gangliosides are biologically relevant molecules during cellular differentiation and development. First, outgrowth of cellular extensions, one aspect of neuronal differentiation, can be induced by treating $\mathrm{NB}$ cells in vitro with exogenous gangliosides, and the $\mathrm{CbG}$ species GT1b and GQ1b have been repeatedly described as the two most potent ganglioside molecules with differentiating properties (Tsuji et al, 1983; Leskawa and Hogan, 1985). Secondly, increased expression of GD1b and GT1b in murine NB cells transfected with GD3 synthase occurs in parallel with morphological signs of increased cellular differentiation (Kojima et al, 1994). Thirdly, absence of all gangliosides downstream of GM2/ GD2 synthase (CbG+GD2 and $\mathrm{CaG}+\mathrm{GM} 2)$ in genetically altered mice with a disrupted gene for that enzyme is associated with decreased myelination and axon degeneration in the central and peripheral nervous system (Sheikh et al, 1999), although brain development and gross behaviour is normal (Takamiya et al, 1996). Finally, although retinoic acid-induced neurite outgrowth is unaffected by abrogation of cellular ganglioside synthesis in LAN-5 cells grown in a serum-supplemented environment (Li and Ladisch, 1997), reduction of cellular ganglioside synthesis is associated with decreased NGF-induced outgrowth of neuritic processes in a NB cell line grown in serum-free medium, and is reversible by exogenous addition of gangliosides (Rosner, 1998).

Previous findings and our demonstration of induction of $\mathrm{CbG}$ (and $\mathrm{CaG}$ ) synthesis by retinoic acid underscore the potential role of complex gangliosides in cellular differentiation and other cellular functions important to reversion of the malignant phenotype. Consequently, the specific role of complex gangliosides compared to structurally simpler molecules in influencing cellular differentiation and other cellular functions in NB and other neuroectodermal tumours will be important to delineate.

\section{ACKNOWLEDGEMENTS}

We thank Dr Patrick Reynolds, Children's Hospital Los Angeles, for kindly providing human NB cell lines SMS-KCNR and SMSKCN. This study is supported in part by National Institutes of Health Grants CA-90362 (KK), CA-42361 (SL) and by The Children's Cancer Foundation, Baltimore, MD. Dr Hettmer was the recipient of a fellowship by the Dr Mildred Scheel Stiftung/ Deutsche Krebshilfe. 


\section{REFERENCES}

Floutsis G, Ulsh L, Ladisch S (1989) Immunosuppressive activity of human neuroblastoma tumor gangliosides. Int J Cancer 43: 6-9

Fukumoto S, Mutoh T, Hasegawa T, Miyazaki H, Okada M, Goto G, Furukawa K, Urano T (2000) GD3 synthase gene expression in PC12 cells results in the continuous activation of TrkA and ERK1/2 and enhanced proliferation. J Biol Chem 275: $5832-5838$

Hakomori S (1996) Tumor malignancy defined by aberrant glycosylation and sphingo(glyco)lipid metabolism. Cancer Res 56: 5309-5318

Hettmer S, Malott C, Woods W, Ladisch S, Kaucic K (2003) Biological stratification of human neuroblastoma by complex ' $b$ ' pathway gangliosides. Cancer Res 63: 7270-7276

Hirschberg K, Zisling R, van Echten-Deckert G, Futerman AH (1996) Ganglioside synthesis during the development of neuronal polarity. Major changes occur during axonogenesis and axon elongation, but not during dendrite growth or synaptogenesis. J Biol Chem 271: 14876-14882

Hynds DL, Summers M, Van Brocklyn J, O’Dorisio MS, Yates AJ (1995) Gangliosides inhibit platelet-derived growth factor-stimulated growth, receptor phosphorylation, and dimerization in neuroblastoma SH-SY5Y cells. J Neurochem 65: $2251-2258$

Kanda N, Nakai K, Watanabe S (2001) Gangliosides GD1b, GT1b, and GQ1b suppress the growth of human melanoma by inhibiting interleukin-8 production: the inhibition of adenylate cyclase. J Invest Dermatol 117: $284-293$

Kanda N, Tamaki K (1998) Ganglioside GQ1b enhances Ig production by human PBMCs. J Allergy Clin Immunol 102: 813-820

Kanda N, Tamaki K (1999a) Ganglioside GD1b supresses immunoglobulin production by human peripheral blood mononuclear cells. Exp Hematol 27: $1487-1493$

Kanda N, Tamaki K (1999b) Ganglioside GT1b suppresses immunoglobulin production by human peripheral blood mononuclear cells. Immunology 96: $628-633$

Kanda N, Watanabe S (2001) Gangliosides GD1b, GT1b, and GQ1b enhance IL-2 and IFN-gamma production and suppress IL- 4 and IL-5 production in phytohemagglutinin-stimulated human T cells. J Immunol 166: $72-80$

Kaucic K, Etue N, LaFleur B, Woods W, Ladisch S (2001) Neuroblastomas of infancy exhibit a characteristic ganglioside pattern. Cancer 91: 785-793

Kemp SF, Stoolmiller AC (1976) Studies on the biosynthesis of glycosphingolipids in cultured mouse neuroblastoma cells: characterization and acceptor specificities of $\mathrm{N}$-acetylneuraminyl- and $\mathrm{N}$-acetylgalactosaminyltransferases. J Neurochem 27: 723-732

Kohl NE, Kanda N, Schreck RR, Bruns G, Latt SA, Gilbert F, Alt FW (1983) Transposition and amplification of oncogene-related sequences in human neuroblastomas. Cell 35: 359-367

Kojima N, Kurosawa N, Nishi T, Hanai N, Tsuji S (1994) Induction of cholinergic differentiation with neurite sprouting by de novo biosynthesis and expression of GD3 and b-series gangliosides in Neuro2a cells. $J$ Biol Chem 269: 30451 - 30456

Ladisch S, Gillard B (1985) A solvent partition method for microscale ganglioside purification. Anal Biochem 146: 220-231

Ladisch S, Kitada S, Hays EF (1987) Gangliosides shed by tumor cells enhance tumor formation in mice. J Clin Invest 79: 1879-1882

Ladisch S, Li R, Olson E (1994) Ceramide structure predicts tumor ganglioside immunosuppressive activity. Proc Natl Acad Sci USA 91: 1974-1978

Ledeen RW, Yu RK (1982) Gangliosides: structure, isolation, and analysis. Methods Enzymol 83: 139-191

Leskawa KC, Hogan EL (1985) Quantitation of the in vitro neuroblastoma response to exogenous, purified gangliosides. J Neurosci Res 13: 539-550

Levine JM, Flynn P (1986) Cell surface changes accompanying the neural differentiation of an embryonal carcinoma cell line. J Neurosci 6: $3374-$ 3384

Li R, Ladisch S (1991) Shedding of human neuroblastoma gangliosides. Biochim Biophys Acta 1083: 57-64

Li R, Ladisch S (1992) Alteration of neuroblastoma ganglioside metabolism by retinoic acid. J Neurochem 59: $2297-2303$

Li R, Ladisch S (1997) Inhibition of endogenous ganglioside synthesis does not block neurite formation by retinoic acid-treated neuroblastoma cells. I Biol Chem 272: 1349 - 1354

Liour SS, Kapitonov D, Yu RK (2000) Expression of gangliosides in neuronal development of P19 embryonal carcinoma stem cells. J Neurosci Res 62: $363-373$

Lovat PE, Lowis SP, Pearson AD, Malcolm AJ, Redfern CP (1994) Concentration-dependent effects of 9-cis retinoic acid on neuroblastoma differentiation and proliferation in vitro. Neurosci Lett 182: 29-32
Matthay KK, Villablanca JG, Seeger RC, Stram DO, Harris RE, Ramsay NK, Swift P, Shimada H, Black CT, Brodeur GM, Gerbing RB, Reynolds CP (1999) Treatment of high-risk neuroblastoma with intensive chemotherapy, radiotherapy, autologous bone marrow transplantation, and 13-cisretinoic acid. Children's Cancer Group. N Engl J Med 341: 1165-1173

Melino G, Thiele CJ, Knight RA, Piacentini M (1997) Retinoids and the control of growth/death decisions in human neuroblastoma cell lines. $J$ Neurooncol 31: $65-83$

Nakagawara A, Arima-Nakagawara M, Scavarda NJ, Azar CG, Cantor AB, Brodeur GM (1993) Association between high levels of expression of the TRK gene and favorable outcome in human neuroblastoma. $N$ Engl J Med 328: $847-854$

Osanai T, Watanabe Y, Sanai Y (1997) Glycolipid sialyltransferases are enhanced during neural differentiation of mouse embryonic carcinoma cells, P19. Biochem Biophys Res Commun 241: 327 - 333

Pohlentz G, Kaes C, Sandhoff K (2000) In vitro assays for enzymes of ganglioside synthesis. Methods Enzymol 311: 82-94

Rebhan M, Vacun G, Bayreuther K, Rosner H (1994) Altered ganglioside expression by SH-SY5Y cells upon retinoic acid- induced neuronal differentiation. NeuroReport 5: $941-944$

Rettig WJ, Spengler BA, Chesa PG, Old LJ, Biedler JL (1987) Coordinate changes in neuronal phenotype and surface antigen expression in human neuroblastoma cell variants. Cancer Res 47: 1383-1389

Reynolds CP, Biedler JL, Spengler BA, Reynolds DA, Ross RA, Frenkel EP, Smith RG (1986) Characterization of human neuroblastoma cell lines established before and after therapy. J Natl Cancer Inst 76: 375-387

Reynolds CP, Lemons RS (2001) Retinoid therapy of childhood cancer. Hematol Oncol Clin North Am 15: 867 -910

Rizzo AM, Gornati R, Rossi F, Bernardini G, Berra B (1995) Retinoic acid induces changes in Xenopus embryo glycolipid pattern. Cell Biol Int 19: $895-901$

Rosner H (1998) Significance of gangliosides in neuronal differentiation of neuroblastoma cells and neurite growth in tissue culture. Ann N Y Acad Sci 845: $200-214$

Schengrund CL, Repman MA, Shochat SJ (1985) Ganglioside composition of human neuroblastomas. Correlation with prognosis. A Pediatric Oncology Group Study. Cancer 56: 2640-2646

Schengrund CL, Shochat SJ (1988) Gangliosides in neuroblastomas. Neurochem Pathol 8: 189-202

Sheikh KA, Sun J, Liu Y, Kawai H, Crawford TO, Proia RL, Griffin JW, Schnaar RL (1999) Mice lacking complex gangliosides develop Wallerian degeneration and myelination defects. Proc Natl Acad Sci USA 96: 7532 7537

Srinivas L, Colburn NH (1982) Ganglioside changes induced by tumor promoters in promotable JB6 mouse epidermal cells: antagonism by an antipromoter. J Natl Cancer Inst 68: 469-473

Sung CC, Pearl DK, Coons SW, Scheithauer BW, Johnson PC, Zheng M, Yates AJ (1995) Correlation of ganglioside patterns of primary brain tumors with survival. Cancer 75: $851-859$

Svennerholm L, Bostrom K, Fredman P, Mansson JE, Rosengren B, Rynmark BM (1989) Human brain gangliosides: developmental changes from early fetal stage to advanced age. Biochim Biophys Acta 1005: 109 117

Takamiya K, Yamamoto A, Furukawa K, Yamashiro S, Shin M, Okada M, Fukumoto S, Haraguchi M, Takeda N, Fujimura K, Sakae M, Kishikawa M, Shiku H, Aizawa S (1996) Mice with disrupted GM2/GD2 synthase gene lack complex gangliosides but exhibit only subtle defects in their nervous system. Proc Natl Acad Sci USA 93: $10662-10667$

Tsuji S, Arita M, Nagai Y (1983) GQ1b, a bioactive ganglioside that exhibits novel nerve growth factor (NGF)-like activities in the two neuroblastoma cell lines. J Biochem (Tokyo) 94: 303-306

Valentino L, Moss T, Olson E, Wang HJ, Elashoff R, Ladisch S (1990) Shed tumor gangliosides and progression of human neuroblastoma. Blood 75: $1564-1567$

van Echten G, Sandhoff K (1993) Ganglioside metabolism. Enzymology, topology, and regulation. J Biol Chem 268: $5341-5344$

Wu ZL, Schwartz E, Seeger R, Ladisch S (1986) Expression of GD2 ganglioside by untreated primary human neuroblastomas. Cancer Res 46: $440-443$

Yates AJ, Franklin TK, McKinney P, Collins R, Comas T, Boesel CP, Pearl DK (1999) Gangliosides and neutral glycolipids in ependymal, neuronal and primitive neuroectodermal tumors. J Mol Neurosci 12: 111-121 